\title{
Parents' Perceptions towards the Role of School Health Units at Early Detection of Ocular Disorders among Students in Qassim Province, Saudi Arabia
}

\author{
Hamad F.Al-Qesair \\ Dr. Abdulrahman Alshammari \\ Department of Public Administration College of Business Administration King Saud University \\ Email: Alshammari1960@hotmail.com
}

\section{Doi:10.5901/mjss.2016.v7n4p}

\begin{abstract}
In Saudi Arabia, vision screenings are usually carried out by school health units (SHUs) and are mandatory for all children enrolling in schools. However, these screenings can miss visual disorders that could lead to public health problems. The purpose of this study was to examine parent perceptions of the role of SHUs towards early detection of visual problems among male students in Qassim Province, Saudi Arabia. The objectives of this research were; (1) to critically identify the relationship between school health units and early detection of visual disorders, as well as the impact of school services and activities on early detection of eye problems from the perspective of students' parents, and (2) to provide recommendations for health policies that may help reduce the prevalence of ocular disorders among students. Descriptive statistics were used to analyses responses of 100 parents from ten schools in two chosen areas in Qassim Province, regarding the role of SHUs and school support, as factors perceived by students' parents associated with early detection of ocular disorders. The findings of the research indicated inequity in accessibility to the SHUs' facilities. Furthermore, there was a lack of vision screenings by a SHU. In addition, the role of the school was not supported in detecting eye problems earlier. This study generally found that vision screenings conducted by SHUs are still insufficient after more than three decades. Accordingly, it was recommended to increase knowledge about eye health care for students and their parents, and to establish health clinics in schools.
\end{abstract}

Keywords: Early detection of ocular disorders, Visual acuity (VA), School Health Unit (SHU), School support, Vision screening.

\section{Introduction}

In many countries, health and education organisations usually work separately with different goals. However, there is an inextricable link between health and education through school health services. School health services contain immunization, screening, surveillance, counseling, early detection and treatment, and referral services (Prasad, 2005). In Saudi Arabia, school health services are managed by the General Administration of School Health (GAOSH), under the supervision of the Ministry of Education (MOE) Today, there are 163 school health units (SHUs) throughout the country. School health services are provided through three levels- prevention, early detection, and dealing with chronic problems. The level of early detection services consists of two important programs; primary mandatory health examinations for preschool children, and a periodic comprehensive health examination. Vision screening is carried out as part of a twoprogram (Alansari, 2001)

In summary, the efficiency of the vision-screening program helps reduce the prevalence of eye diseases. Therefore, this study aims to identify the role of SHUs in detecting visual disorders among preschool children, or primary and intermediate students from the perspective of students' parents in Qassim Province, Saudi Arabia.

\subsection{Statement of the Problem}

In Qassim Province, school health units for both genders concentrate on the province's cities, and are responsible for providing health services to the hundreds of male and female schools. Vision screening for preschool children is usually implemented by using the Logarithm of the Minimum Angle of Resolution (log MAR) charts or Snellen Visual Acuity (SVA) charts. They are useful in examining visual acuity (VA), and near and distant vision (General Administration of School Health, 2007).

In many schools, teachers are often able to detect eye problems, such as visual impairment, color vision, or near and distance vision. The published data on the prevalence of ocular disorders among preschool children in Qassim 
indicates the severity of late detection of eye problems. For example, (Qureshi, Ahmed, Al-debasi, \& Nair, 2012) found there were 203 students (14.36\%) with refractive problems. Also, (Aldebasi, 2014) revealed that the prevalence of correctable refractive error was $18.6 \%$. Finally, vision screening of SHU is held prior to entering school; as well as periodic screenings. However, these screenings can miss vision disorders that could lead to a public health problem. Due to the intensity of the issue, there is a need to conduct a study to identify the role of school health units (SHUs), as well as schools in early detection of correctable visual problems for male preschool children and students in two chosen areas of Qassim Province, Saudi Arabia.

\subsection{Research Objectives}

The research objectives of this study are as following:

1. To identify the relationship between school health units and early detection of visual disorders from the parents' perspective;

2. To identify the impact of schools' services and activities on early detection of eye problems;

3. To bring recommendations to health decision makers that may help reduce the prevalence of ocular disorders among students.

\subsection{Research Questions}

R.Q. 1: What is the overall perspective of students' parents towards the ability of school health Units? In detecting eye problems earlier

R.Q. 2: Which is the impact of schools' services and activities on early detection of eye problems?

R.Q. 3: What are ways to help manage ocular disorders among students?

\subsection{Significance of the Study}

In Saudi Arabia, the problem of vision screening in early detection has not received a great deal of attention. Nearly all previous studies have been focused on the prevalence of eye problems. This research will be unique because it addresses an under-researched area pertaining to the role of vision screenings for preschool children and students at early detecting of visual problems that may be treated and prevented the prevalence.

In the community, school students fall into the preventable age group. This is an important opportunity to identify the role of vision screening in decreasing ocular disorders. Undoubtedly, the efficiency of vision screening of school health units (SHU) leads to early detection. (Fryer, Igoe, \& Miyoshi, 1997) found that basic visual acuity screening at a school is useful for early detection of possible vision problems. Local studies emphasized the importance of periodic vision screenings in schools at early detecting. Based on this, it would be interesting to explore the role of school health units (SHUS's), including sufficient vision screenings and related services, as well as the role of the school's activities and services at early detection of eye problems.

In short, results of this study will reveal the factors correlated with the late detection of eye problems. As a result, decision makers might be able to tailor their decisions to most efficiently address correctable ocular disorders. The depth of this study will aid future implementation and enforcement of vision screenings with continuing improvement in order to decrease ocular disorders among male children in Qassim Province, Saudi Arabia.

\subsection{Definitions of Key Terms}

- Early detection of ocular disorders: Is the ability of the vision screening of school health units and Early diagnosis of eye problems.

- Visual acuity (VA) in school students: refers to the sharpness of student eyesight. Visual acuity in School students is an outcome from vision screening of all aged-students, particularly in primary and Intermediate grades.

- School Health Unit: is the facility responsible for conducting vision screening among students or preschool children, through a periodic comprehensive health examination or mandatory health examinations for school enrollment.

- School support: refers to health services and activities supplied to students. The main goal of school support is to educate and help those in need of health prevention and promotion, including ocular disorders. 
- Vision screening: a limited series of examinations to identify visual impairment or other ocular abnormalities in students or preschool children.

\subsection{Importance of School Health Services}

School health is considered a public health mission carried out by the school health centers or units. There is consensus among public health researchers that the health of children and youth is a fundamental value. Therefore, schools are an ideal environment for providing their health services. In general, school health centers provide the basic tools of primary and preventive care; such as vision and hearing screening programs (Harvey, Vaquerano, Nolan, \& Sonosky, 2002). One of the school health services functions is to provide preventive and acute care (American Academy of Pediatrics, 2001). In specific cases, Santelli, Kouzis, and Newcomer (1996) found that using of school health centers decreases the overuse of emergency departments. Also, (Kisker \& Brown, 1996) found that the using school health services increased student access to health care and improved their health knowledge.

However, previous research has shown that many challenges faced delivery of school health services. For example, Jen (2009) revealed that school health centers have insufficient resources to provide follow-up healthcare for students. In another study, Lear (2002) found that because of absent school nurses, a large number of sick or injured students would be transported to emergency rooms. So, a hospital is obliged to respond to community need.

\subsection{Importance of Vision Screening for School Students}

Vision screening is definitely responsible for early detection of many ocular disorders. Conducting periodic vision screenings is a cost effective method for early detection of visual impairment in children and should be done at preschool and school entry level (Aldebasi, 2014; Qureshi et al., 2012). Also, WHO's global initiative "Vision 2020" recommended that the vision screening program for all school-aged children should provide early detection and initiate early treatment (Al-Rowaily, 2010). Accordingly, a considerable amount of literature has been published on the importance of vision screening for school students. It was shown by Resnikoff, Pascolini, Mariotti, and Pokharel (2008) that visual impairment from uncorrected refractive errors can have negative effects in terms of lost education and future lost employment opportunities, which may influence quality of life. So, the study of Taylor, Pezzullo, Nesbitt, and Keeffe (2007) showed that much can be done to prevent vision loss that is cost effective. Powell, Wedner, and Richardson (2005) reported that in normal visual development, changes in refractive error occur in the first few years of life. Undoubtedly, vision screening of preschool age and school children is a fundamental priority in detecting visual disorders, and preventing or correcting them at the appropriate time (Al Wadaani, Amin, Ali, \& Khan, 2013; Bardisi \& Bin Sadiq, 2002).

Several studies have focused on sufficient vision screenings for students in KSA. Although preschool visual screening in Saudi Arabia aims to early identify children with visual problems, students were just screened for distance vision (Al Wadaani et al., 2013; Rowaily \& Alanizi, 2010). Some studies have revealed that the current vision screenings of the school health units (SHUs) are still insufficient. Bardisi and Bin Sadiq (2002) stated that the Saudi school health services provided by the ministry of education do not have adequate vision screening programs, and often are not carried out properly. This finding is consistent with finding of the past study by (Al-Nasser, Sinha, \& Ali, 1989) which noted there is a gross lack of eye examination in different parts of rural KSA.

To ensure early treatment and intervention, a number of studies have examined barriers to obtaining eye care services, particularly after failed visual acuity screenings. These studies focused on the follow-up, accessibility and referral services of vision screening. Su et al. (2013) found that miscommunication of visual acuity screening failure was the main reason for not obtaining follow-up. Those who failed to obtain follow-up claimed that they had not been informed of their child's screening failure. Al Wadaani et al. (2013) in contrast found that although the reported better access into eye care services in Al-Hassa, KSA, the prevalence of visual impairment from uncorrected refractive errors in some regions appears to be higher in urban areas than rural areas. Screening can enable important health problems to be identified earlier, offering effective interventions, but it is futile if follow-up or treatment is unavailable (Mathers, Keyes, \& Wright, 2010). In developing countries, the majority of children in rural areas never receive an eye examination and have limited access to health services (Wedner, Ross, Balira, Kaji, \& Foster, 2000; Wedner. S. \& Dineen. B., 2003).

In order to increase the accessibility-level to school-based follow-up and referral services of vision screening, Aldebasi (2014) called for implementing child eye health programs that could be integrated within school health programs. Also, Basch (2010) recommended that school health service practitioners or school health program coordinators should establish a referral network of vision care services in the community.

Researchers also determined that numerous factors are associated with late detection of vision abnormalities. This 
is apart from these findings related to potential negative impacts of eye examination tools, and the role of school support in early detection. Hinkley, Abata, and Schlotthauer (2014) revealed that teachers are the first to notice vision problems in primary-aged children, as they spend the majority of the working day with the child. So, Gupta, Lal, Mazta, and Sharma (2012) stated that training and periodic supervision of vision screening by school teachers could improve the accuracy of the vision test. The validity of eye examination tools such as Snellen charts are helpful in a perfect diagnosis, but Fotouhi, KhabazKhoob, Hashemi, Yekta, and Mohammad (2011) found that the application of Snellen charts in screening fails to identify hyperopic cases. In fact, schools have a significance role in handling the problem of late detection of vision problems. Basch (2010) mentioned that although many vision problems are preventable if the diagnoses are provided appropriately, results of school vision screening programs depend on the instruments and methods utilised. He added that the main roles of teachers are to help children with learning-related vision problems. Accordingly, teachers and school health-care workers should receive training and information programs (Resnikoff et al., 2008).

\subsection{The Relationship between Vision Screening and Prevalence of Ocular Disorders in Saudi Arabia}

A number of studies have investigated the prevalence of eye problems among students in Saudi Arabia. These studies showed scare data about ocular disorders across the country, Recent data reported a prevalence of refractive errors among school children, from the studies conducted. In Qassim Province Aldebasi (2015) it was 3.90\%, and the prevalence of amblyopia in this province (Aldebasi, 2014) was 18.6\%; Taif City (Desouky, Murad, \& Khan, 2014) was 16.4\%; Al-Hassa Province (Al Wadaani et al., 2013) was13.7\%; Buraidah City (Qureshi et al., 2012) was 14.36\%; Riyadh City (Al-Rowaily, 2010) was 4.5\%; also Riyadh City (Rowaily \& Alanizi, 2010) was 9.8\%; and Jeddah City (Bardisi \& Bin Sadiq, 2002) was $69 \%$ from 50 cases

Interestingly, previous local studies agreed that the lack of vision screening by school health unit contributed to increasing the incidence of vision abnormalities. These studies emphasised the importance of vision screenings. Research findings by Desouky et al. (2014) showed that the prevalence of refractive errors warrants integration of visual screening for refractive error in school health programs. Consequently, Rushood et al. (2013) reported that the prevalence of eye diseases in schoolchildren might be raised because of an absence of an efficient school screening program. Qureshi et al. (2012) showed that the incidence of undiagnosed amblyopia suggests screening should be conducted at the preschool and school entry level. Therefore, Bardisi and Bin Sadiq (2002) stated that the way to decrease the incidence of amblyopia is early detection at preschool age. Importantly, recent data about the prevalence of ocular disorders can help decision makers design, implement, and evaluate screening programs. Aldebasi (2015) suggested that data from the prevalence studies will aid the ministry of health in designing a strategy for effective screening.

\subsection{Knowledge of Eye Health}

Knowledge of eye health enables teachers, and students and their parents to recognize ocular problems, and then motivates them to utilise eye care services including vision screenings, comprehensive eye examinations, and other services. Some previous studies investigated the importance of visual health awareness in decreasing the incidence of common eye diseases among students, which can lead to visual impairment; whereas a handful of studies examined awareness of eye care services.

Firstly, in regards to visual health awareness, recent studies indicated a lack of awareness about visual health in a school community. Arif and Mehboob (2014) showed that children do not complain of visual problems, and due to lack of awareness, these complaints remained unnoticed by the parents and led to ocular disorders. Also, Nila Ethel and Nagalakshmi (2014) pointed out the major cause of a high prevalence of refractive error is parent and teacher ignorance in identifying refractive error. Another study byWoodhouse, Davies, McAvinchey, and Ryan (2014) found that some children have a visual difficulty of which parents and teachers are unaware. Thereby, Nila Ethel and Nagalakshmi (2014) recommended that educating parents and teachers through the media or awareness programs is necessary and should be carried out by government and non-governmental organizations. Similarly, Aldebasi (2014) recommended educating parents and teachers about refractive error, and about the need for early screening and intervention. Also, the findings of Desouky et al. (2014) study emphasized the necessity of health education sessions for students and parents in order to increase awareness of risk factors of refractive errors.Gursoy, Basmak, Yaz, and Colak (2013) mentioned that vision screening in children is very important because many children are often unaware that their sight may be worse than normal. Al Wadaani et al. (2013) recommended that school teachers, children and parents should be educated about signs and symptoms of refractive error and for the risk factors involved in its development. Consequently, Aldebasi (2011) 
stated that awareness of eye problems and treatment can play an important role in the prevention of blindness from common eye disorders. The finding by (Fotouhi et al., 2011) study discussed that some hypermetric students, are unaware of their condition. Also, Resnikoff et al. (2008) reported that one of the factors for refractive errors remaining uncorrected is lack of awareness and recognition of the problem at the personal, family, community and public health level. In Saudi Arabia, there is only one study by (Aldebasi) that examined awareness of visual health. It found that although $86 \%$ of those surveyed knew about the two most common types of refractive errors (farsightedness, nearsightedness), and is not aware of most of the problems-related to their visual health. To enhance the level of eye problem awareness, Murthy (2000) reported that school vision testing programs have an impact on concerned communities by increasing their knowledge of vision disorders and how to manage them.

Secondly, knowledge of the school health facility site and the availability of eye care services could enable students to access to these services when needed. (Al Wadaani et al., 2013; Rowaily \& Alanizi, 2010) agreed that the prevalence of refractive errors among students warranted affordability and accessibility of corrections for everyone. They recommended facilitating education and awareness campaigns to ensure the corrections are used, and cultural barriers to compliance are addressed and removed. However, Baltussen, Naus, and Limburg (2009) cleared that access to eye care services, and public awareness of their need has not yet reached adequate levels. This is consistent with findings from previous studies in Saudi Arabia. For example, Alotaibi (2006) revealed that the lack of low vision specialists is one of the important reasons why Saudi society has not been aware of the significance of low vision service. Kemper, Uren, and Clark (2006) also found that the greatest barriers of preschool-aged children for compliance in follow-up eye care were a lack of knowledge about the benefits of early intervention. Furthermore, Kimel (2006) reported that parent perceptions of vision problems were significant barriers.

\section{Research Method and Design}

The primary method in this research is a quantitative study, while its design is a descriptive correlational analysis. The study has two independent variables- vision screenings conducted by SHUs and school support. The two variables may directly influence the dependent variable, which is early detection of ocular disorders. These variables were drawn from literature of the vision screening for preschool children, and the pilot study conducted before the main survey of the study. Accordingly, this design is made to analyse the impact of the vision screenings conducted by school health units for early detection of some eye problems among students in Qassim Province, Saudi Arabia.

\subsection{Study Population}

Since the focus of this research is on male school health units, the targeted population is focused on male primary and intermediate students who have never screened their vision through these units before or after entering schools. This research will be focused on responding to the student's parent or relatives.

\subsection{Study Sample}

This study employed a self-administered questionnaire. It was carried out in ten male schools (Five primary grades and five intermediate grades) from two chosen areas in Qassim Province. A stratified random sampling procedure was used to collect data from students' parents or relatives whose sons are presently studying at targeted schools. The sample selected 10 students from five primary schools, and the same number was also selected from five intermediate schools. The priority of the overall sample (100 students) was focused on those with eye problems, according to the students health problems list available by the students advisor of each school. The schools' administrative offices sent the questionnaires to the students' parents, informed them about the study, and encouraged them to fill out the questionnaire. The total numbers of distributed questionnaires were 100 (for each school, 10 questionnaires). Among the questionnaires that were distributed, a total of 83 participants $(n=83)$ replied. Thus, the returned questionnaires were valid for analysis, with an acceptable response rate of $83 \%$.

\subsection{Instrument}

The research is using a primary survey questionnaire designed with closed-ended structured questions (Arabic and English versions) to determine the perceptions of primary and intermediate students' parents regarding the child's age, knowledge of eye health, vision screenings of school health units, and school support; as associated factors with early 
detection of ocular disorders among students in Qassim Province. Therefore, the questionnaire is designed to elicit information on different variables that serve the purpose of the study. The title and purpose of the study are outlined in the cover of the questionnaire's form. It comprises different questions; including multiple choices, multiple responses, dichotomous (Yes/No) questions, and Likert scale.

To increase the content validity of the questionnaire, the study's variables were carried out and selected according to the review of the relevant literature; In addition, it was pre-tested in a pilot survey of 10 respondents to ensure that the wording, format, length, and range of the scales were appropriate. Furthermore, an academic panel of tow staff reviewed the questionnaire and made comments and suggestions. Based on the outcome of the pilot study, a few questions were reformulated, and others were added and excluded. The pilot survey questionnaires were not included in the main study. The reliability of the questionnaire was measured by using the Cronbach's alpha, which was found to be $80.8 \%$.

The English version was translated into Arabic by the researcher. Specialists in English, who were interested in Arabic, reviewed the translated version. The specialists have considered the clarity of the questions and cultural adaptation.

\section{Data Collection and Analysis}

The questionnaires were distributed among 10 male school students in two cities of Qassim province, including rural areas; from the 12 to 23 April 2015. After data was collected, it was entered and analysed by using the Statistical Package for the Social Sciences (SPSS, Version 20); using a descriptive analysis (particularly, frequencies and percentages).

Non-parametric inferential statistics were performed, using Chi-Square test (Cramer's V coefficient) and Spearman Rho test. Cramer's V coefficient is useful to test differences between two nominal variables; such as detecting a visual problem and students who have a visual problem; while Spearman Rho is carried out to determine correlation coefficients between two ordinal dimensions; such as coordination for vision screenings between School \& SHU and referring eye problem cases from school to SHU. Performing these statistics tools are useful to detect if there is a significant relationship between school health units as well as schools with early detection of eye problems. $5 \%$ is taken on the critical significance level of test statistics.

\subsection{Descriptive Statistical Analysis:}

This section presents the analysis of descriptive statistical results regarding the student's age, knowledge of eye health care, vision screenings of school health units, and school support as associated factors in early detection of ocular disorders among students in Qassim Province. Table 1 shows the majority of the participants fell in the age groups 10-12 and $13-15$ (31.3\% and $32.5 \%$ respectively).

Table 1: Descriptive Analysis of Student Age Group

\begin{tabular}{|c|c|c|c|}
\hline Variable & Age Group & Freq. N= 83 & $\%$ \\
\hline & $<6$ & 2 & 2.4 \\
\multirow{3}{*}{ Student's age } & $6-9$ & 13 & 15.7 \\
& $10-12$ & 26 & 31.3 \\
& $13-15$ & 27 & 32.5 \\
& $>15$ & 15 & 18.1 \\
\hline
\end{tabular}

Table 2 shows participants' responses on their knowledge of eye health care. It was found that the majority of the participants (74.7\%) knew the location of SHU. It is also found that 39 (47\%) of parents' students knew that SHU provides general eye exams, and the same number of the sample responded that they did not know about the services that are provided by SHU. For visual acuity and eye examinations, it was found that the vast majority of the participants did not differentiate between them. 65 (78.3\%) of parents' students knew that eye diseases were as important as other diseases, whereas 18 of them (10.8\% and $10.8 \%$ respectively) did not have adequate knowledge of the significance of eye diseases. It was found that 36 (43.4\%) of the whole sample knew that wearing spectacles corrects all eye problems, and almost the same percentage of them (41\%) responded that wearing spectacles does not benefit in correcting all eye problems. 
Table 2: Descriptive Analysis of Eye Health Care Knowledge

\begin{tabular}{|l|c|c|c|}
\hline Variable & Item & Freq. N= 83 & $\%$ \\
\hline Knowledge of SHU location & Yes & 62 & 74.7 \\
& No & 21 & 25.3 \\
\hline \multirow{2}{*}{ Knowledge of SHU eye services } & General eye exams & 39 & 47.0 \\
& Eye disease treatment & 5 & 6.0 \\
\hline \multirow{2}{*}{ Differentiate between visual acuity \& eye exam } & Don't know & 39 & 47.0 \\
\hline \multirow{2}{*}{ Knowledge of eye diseases Importance } & Yes & 25 & 30.1 \\
& No & 17 & 20.5 \\
& Don't know & 41 & 49.4 \\
\hline \multirow{2}{*}{ Knowledge of wearing spectacles benefits } & Yes & 65 & 78.3 \\
& No & 9 & 10.8 \\
& Don't know & 9 & 10.8 \\
\hline & Yes & 36 & 43.4 \\
& No & 34 & 41.0 \\
\hline
\end{tabular}

Table 3 presents perceptions of the student's parents on the role of SHU at detecting ocular disorders in the early stages, as they had an experience of vision screening in a SHU. Regarding the first experience of vision screening, it showed that more than half the participants (59\%) had their first experience in a SHU at a mandatory screening for preschool enrollment; and (41\%) before enrolling in school. Considering the health facility which the participants had accessed to conduct the first vision screening, the majority of the participants (65.1\%) had not accessed the SHU. It was found that 29 of the participants had used a SHU, 19 a primary healthcare center (PHC), and 35 a private health facility $(34.9 \%$, $22.9 \%$ and $42.2 \%$ respectively). The main reason prevented 54 of the participants (nearly $65 \%$ ) from visiting a SHU. It was found that the reason of distance from the participants' residence was the highest percentage among the rest of reasons ( $n=25,30.1 \%)$. As regards of the periodic vision screenings, this study revealed that the vast majority of participants ( $n=72,86.7 \%$ ) were not informed about conducting periodic vision screenings at school by SHU in the past two years. The vast majority of participants felt that vision screenings conducted by SHU were not sufficient in detecting eye problems when $76(80.7 \%)$ of them responded no. With respect to eye problems, it was found that the majority of the students ( $n=52,62.7 \%)$ had problems with their eyes; and $43(51.8 \%)$ of them wear spectacles. It is not surprising when the study found that $40(48.2 \%)$ of the students with eye problems were detected by their families not by a SHU. This was because it showed there were a few follow-up and referral procedures (14.4\%) which were done by SHU after detecting these problems. To conclude the role of SHU at early detection of eye problems, the participants rated these services as their sons $(n=52,62.7 \%)$ had eye problems, and accessed SHU's eye services. It is found that although there was a poor rating of eye services $(n=24,28.9 \%)$, there was an acceptable rating between good and fair $(n=15,18.1 \%$ and $n=13$, $15.7 \%$ respectively); and formed the percentage of (33.8\%).

Table 3: Descriptive Analysis According the Role of SHU at Early Detection

\begin{tabular}{|l|c|c|c|}
\hline Variable & Item & Freq. N= 83 & $\%$ \\
\hline \multirow{2}{*}{ First vision screening } & Before school enrollment & 34 & 41.0 \\
& At a mandatory screening & 49 & 59.0 \\
\hline \multirow{3}{*}{ Health facility of taking first vision screening } & SHU & 29 & 34.9 \\
& PHC & 19 & 22.9 \\
& Private & 35 & 42.2 \\
\hline \multirow{3}{*}{ Main reason for not visiting SHU } & No responding * & 29 & 34.9 \\
& Far distance & 25 & 30.1 \\
& Material weakness & 6 & 7.2 \\
& Human resources weakness & 10 & 12.0 \\
\multirow{2}{*}{ SHU conducted vision screenings in the past 2 years } & Never heard of SHU & 13 & 15.7 \\
\hline \multirow{2}{*}{ SHU conducted Sufficient Vision Screenings } & Yes & 11 & 13.3 \\
& No & 72 & 86.7 \\
\hline \multirow{2}{*}{ Students who have visual problems } & Yes & 16 & 19.3 \\
& No & 67 & 80.7 \\
\hline
\end{tabular}




\begin{tabular}{|l|c|c|c|}
\hline Variable & Item & Freq. N= 83 & \% \\
\hline \multirow{3}{*}{ Students who wear spectacles } & No responding * & 31 & 37.3 \\
& Yes & 43 & 51.8 \\
& No & 9 & 10.8 \\
\hline & No responding * & 31 & 37.3 \\
Place of detecting a visual problem & A mandatory screening & 2 & 2.4 \\
& Periodic screenings & 4 & 4.8 \\
& A school & 6 & 7.2 \\
Procedures done by SHU after eye's problem detecting & A family & 40 & 48.2 \\
& No responding * & 31 & 37.3 \\
& follow-up appointment & 2 & 2.4 \\
& Referring & 10 & 12.0 \\
SHU's eye services rate & Not detected by SHU & 40 & 48.2 \\
\hline
\end{tabular}

* No responding according to the questionnaire instructions

Table 4 shows the perceptions of the student's parents on the contribution of school with SHU in providing activities and services that help detect eye problems earlier. Responses were gathered from the participants on a five-point Likert scale. According to the Likert scale, the highest percentage of the responses ( $n=57,68.7 \%)$ reported that schools never communicated with parents regarding the coordination for vision screenings with SHU, and no responses reported on always scale. Regarding attending health education sessions, the highest percentage of the responses $(n=64,77.1 \%)$ reported that a school never invited them to attend health education sessions, and no responses reported on always and often scales. With respect to the school communication with parents in regards of discovering eye problems, it was found that 29 of the participants (34.9\%) reported that a school never informed them when eye problem cases were occurring. In addition, if eye problem cases occurred, the study showed that 35 of the participants (42.2\%) reported that a school never referred eye problem cases to the SHU, however, 18 participants (21.7\%) were sometimes reported. Regarding the school health clinic, the study found that (89.2\%) of the responses agreed on that a school health clinic will help in detecting eye problems earlier.

Table 4: Descriptive Analysis According to the Role of School Support at Early Detection

\begin{tabular}{|l|c|c|c|}
\hline Variable & Item & Freq. $\mathbf{N =} 83$ & $\%$ \\
\hline \multirow{4}{*}{ Coordination for vision screenings with SHU } & Always & - & - \\
& Often & 4 & 4.8 \\
& Sometimes & 7 & 8.4 \\
& Rarely & 15 & 18.1 \\
& Never & 57 & 68.7 \\
\hline \multirow{4}{*}{ Invites parents to attend health education sessions } & Always & - & - \\
& Often & - & - \\
& Sometimes & 4 & 4.8 \\
& Rarely & 15 & 18.1 \\
& Never & 64 & 77.1 \\
\hline \multirow{3}{*}{ Informs parents in case of student's eye problem } & Always & 9 & 10.8 \\
& Often & 11 & 13.3 \\
& Sometimes & 16 & 19.3 \\
& Rarely & 18 & 21.7 \\
& Never & 29 & 34.9 \\
\hline \multirow{3}{*}{ Refers eye problem cases from school to SHU } & Always & 6 & 7.2 \\
& Often & 8 & 9.6 \\
& Sometimes & 18 & 21.7 \\
& Rarely & 16 & 19.3 \\
& Never & 35 & 42.2 \\
\hline & Strongly Agree & 61 & 73.5 \\
& Agree & 13 & 15.7 \\
& Neural & 5 & 6.0 \\
& Disagree & 2 & 2.4 \\
& Strongly Disagree & 2 & 2.4 \\
\hline
\end{tabular}




\subsection{Inferential Statistical Analysis}

In order to explore significant relationships between the study variables, the inferential statistics has been performed using Chi-Square test (Cramer's V coefficient) and Spearman Rho test. Cramer's V coefficient was used to test differences between two nominal variables of a SHU and their relation to early detection of ocular disorders, while Spearman Rho was used to determine correlation coefficients between two ordinal variables of a school's activities and services and their relation to early detection of eye problems. The critical significance level of test statistics was considered at $5 \%(p<0.05)$.

Table 5: Cramer's V Coefficient results of the relationship between SHU and Early Detection

\begin{tabular}{|l|c|c|c|}
\hline Variables (nominal by nominal) & & Value & Approx. Sig. \\
\hline The main reason for not visiting SHU \& & Phi & .514 & .038 \\
The rating of the SHU's eye services & Cramer's V & .297 & $.038^{*}$ \\
\hline The main reason for not visiting SHU \& & Phi & .514 & .038 \\
The rating of the SHU's eye services & Cramer's V & .297 & $.038^{*}$ \\
\hline A place of detecting a visual problem \& & Phi & 1.452 & .000 \\
The procedures done after eye problem detection & Cramer's V & .839 & $.000^{*}$ \\
\hline A place of detecting a visual problem \& & Phi & 1.059 & .000 \\
The rating of the SHU's eye services & Cramer's V & .611 & $.000^{*}$ \\
\hline
\end{tabular}

* $p<0.05$

Table 5 shows the result of Cramer's V Coefficient test of the relationship between SHU and early detection of ocular disorders. It was found that there was a relationship between detecting a visual problem and students who have a visual problem $(0.00<0.05)$, and this relation was strong; as Cramer's value was $(+1)$. However, as mentioned in the descriptive analysis (table 3), the majority of eye problems were not detected by a SHU. Regarding the relationship between the main reason for not visiting a SHU and the rating of the SHU's eye services, the study found there was a relationship between these variables $(0.038<0.05)$. However, this relation was not strong as Cramer's value was $(0.3)$ and far away from (+1). This study also found a relationship between detecting a visual problem and the procedures after eye's problem detection $(0.000<0.05)$. While this relation was strong; when Cramer's value was $(0.84)$ and near to $(+1)$, the procedures by a SHU were few; as mentioned in the descriptive analysis (Table 3). The result of the relationship between detecting a visual problem and the rating of the SHU's eye services showed that there was a relationship between detecting a visual problem and the rating of the SHU's eye services $(0.000<0.05)$. However, this relation is fair; because Cramer's value was (0.61).

Table 6 shows the Spearman Rho results of the relationship between school support and early detection of eye problems. The study found that there was a relationship between a two-variable $(0.003<0.05)$. However, this relation is not strong; because a correlation coefficient $(0.32)$ was far from $(+1)$. With respect to the relationship between coordination for vision screenings between school \& SHU and a school informing parents about eye problems, it was found there was a relationship $(0.006<0.05)$. However, this relation is also not strong; when a correlation coefficient $(0$. 30) was far from (+1). It is also found that there was a relationship between a school communicating with parents regarding coordinating vision screenings with a SHU and referring eye problem cases from school to a SHU $(0.014<0.05)$. However, this relation is weak; because a correlation coefficient $(0.27)$ was far from $(+1)$. Regarding the relationship between a school informed parents about eye problems and referring eye problem cases from school to a $\mathrm{SHU}$, it was found there was a relationship $(0.000<0.05)$. This relation is fair when a correlation coefficient $(0.56)$ was far from $(+1)$.

Table 6: Spearman's Rho $(\boldsymbol{\rho})$ Coefficient results of the relationship between School Support and Early Detection

\begin{tabular}{|l|c|c|}
\hline Variables (ordinal by ordinal) & $\boldsymbol{\rho}$ & Sig. (2-tailed) \\
\hline Coordination for vision screenings with SHU \& Health education sessions & $.322^{* *}$ & $.003^{*}$ \\
\hline Coordination for vision screenings with SHU \& School keeps parents informed & $.299^{* *}$ & $.006^{*}$ \\
\hline Coordination for vision screenings with SHU \& Referring to SHU & $.268^{* *}$ & $.014^{*}$ \\
\hline School keeps parents informed \& Referring to SHU & $.560^{* *}$ & $.000^{*}$ \\
\hline
\end{tabular}

${ }^{*} p<0.05{ }^{* *}$ values between $-1 \&+1$ shows the association strength 


\section{Discussions}

This chapter critically examines and discusses the findings with respect to research objectives and their corresponding research questions. The purpose of this research was to examine the impact of the vision screenings conducted by school health units for early detection of some eye problems among students in Qassim Province. The research objectives and questions have been answered from the analysis of results, and showed to what extent they were consistent with previous findings related to vision screening towards early detection of eye problems among students.

The first research question and objective of this research was to identify the relationship between school health units and early detection of visual disorders from the perspective of parents. Discussion of the first question's results begins with first experience of a vision screening by the participants' children. The study found that although $59 \%$ of the participants had their first vision screening at a mandatory screening for preschool enrollment, the majority (65.1\%) had not accessed the SHU. This finding is consistent with findings of the past study by Wedner et al. (2000), which found that the accessibility of the majority of children to health services in developing countries was often limited. In contrast, it is inconsistent with findings by Al Wadaani et al. (2013) which found the age group 5-15 had better access to eye care services in Al-Hassa, KSA. It was found that the main reason for not accessing the SHU was the distance from the parents' residence. In addition, this study indicates a lack of vision screenings by a SHU. It is found that the vast majority of the participants $(86.7 \%)$ were not informed about conducting periodic vision screenings at school by a SHU during the past two years, and (80.7\%) of them were felt that vision screenings conducted by SHU were not sufficient. The present findings agree with previous local studies of (Al-Nasser et al., 1989; Bardisi \& Bin Sadiq, 2002) indicating there is a gross lack of eye examination in different parts of Saudi Arabia, and the Saudi school health services provided by the Ministry of Education does not have adequate vision screening programs. The results also found that the majority $(n=52,62.7 \%)$ from the whole sample have eye problems. Accordingly, this finding indicated there was a strong relationship between those who were able to detect a visual problem and a student with a visual problem. Interestingly, this correlation is related to a negative relation, because families detected the majority of eye problems. Furthermore, the study found a strong relationship between detect a visual problem and the procedures after eye's problem detecting. This finding was not surprising as the procedures by a SHU were few, as eye problems were not detected by a SHU. With regards to the availability of the SHU's eye health services to those who have visual problems, it was found that although there was a poor rating of eye services (28.9\%), there was an acceptable rating between good and fair (33.8\%). This finding indicated a moderate relationship between those who can detect a visual problem, which was a family, and rating the SHU's eye services. In addition, from rating the availability of SHU's eye health services, the study indicated a weak relationship between the main reason for not visiting SHU and rating the SHU's eye services. Among the plausible explanations for these findings is the need to conduct periodic vision screenings by SHUs among students in Qassim province. In addition, a network of vision care services between schools and SHUs should be established to ensure earlier referral and follow-up of any health problems, including ocular disorders.

The second research question and objective of this research was to identify the impact of school services and activities on early detection of ocular disorders. In terms of activities, this study found that schools did not communicate with the majority of the participants regarding coordination of vision screenings with a SHU. In addition, this study revealed that schools never invited the majority of participants to attend health education sessions. This situation revealed the neglect in school activities involving early detection of eye problems. With services provided by schools, despite the substantial differences in responses about being informed by a school when health problems occurred including eye problem cases, the present study found most of the participants ( $n=29,34.9 \%)$ were not informed. In addition, the study found that nearly half of participants reported these cases were not referred to the SHU. Furthermore, this study provides evidence that school support was not strong enough in detecting visual problems earlier. It found there was a weak relationship between a school communicating with parents on coordinating vision screenings with a SHU and inviting them to attend health education sessions. Additionally, there was a weak relationship between a school communicating with parents about coordinating vision screenings with a SHU and informing them about eye problem cases. In addition, the relationship between a school communicating with parents regarding the coordination for vision screenings and referring eye problem cases from school to SHU was weak. Finally, there was also a weak relationship between a school informing parents about eye problems and referring eye problem cases from school to a SHU. These findings support the study of Gupta et al. (2012) which suggested that training and periodic supervision of vision screening by school teachers could improve the validity of the vision test.

The third objective and research question of the present study was to address recommendations to health decision makers that may help reduce the prevalence of ocular disorders among students. Suggestions for preventing or decreasing the prevalence of correctable visual problems among students in this study were approached by examining 
the knowledge of eye health with the age of students, and the value of the school health clinic. Actually, this can help draw realistic implications. The finding of student age showed that the majority of the participants fell in the age groups 10-12 and 13-15. Although some eye problems can be corrected among this type of age-category, this study found that their parents had a lack knowledge of eye health. It is found that nearly half of the participants were not known about the services that are provided by a SHU, while the other half were known that these units just provide general eye exams. In addition, although the vast majority of the participants were known about the importance of eye diseases, nearly half did not know about the benefits of wearing spectacles. That is not surprising when they had a lack of knowledge in eye health, because the majority of the participants in this study have just taken their first vision screening only at a mandatory screening for preschool enrollment. This means that the lack of eye health knowledge among participants in this study had a negative impact concerning visual abnormalities. These findings reflect the finding of Nila Ethel and Nagalakshmi (2014) who indicated the major cause for high prevalence of refractive error is ignorance of parents and teachers in identifying refractive error, and Baltussen et al. (2009) who showed that public awareness of the need for eye care services has not yet reached adequate levels. Therefore, it is apparent that increasing knowledge of eye health services provided by SHUs will be a pragmatic solution of the prevalence of ocular disorders among students. Ultimately, this study highlighted the importance of a school health clinic in preventing most of eye problems among students, when the vast majority of the participants $(n=77,89.2 \%)$ agreed that a school health clinic will help in eye problem detection earlier. In accordance with this finding, a school health clinic will be considered as another practical solution to decrease or prevent eye problems.

\section{Conclusion}

The present study was designed to determine the impact of vision screenings in early detection of some eye problems among students in Qassim Province, Saudi Arabia. This impact was determined by examining the parents' perceptions of the role of SHUs, as well as school support in early detection. It has found that generally, the role of SHUs were not strong enough to decrease the prevalence of eye problems among students in this province.

One of the more significant finding to emerge from this study was that the majority of participants had not accessed the school health unit (SHU) in their first experience of vision screening, because these units were too far away from the parents' residence. The most obvious finding to emerge from this study was that there was a lack of vision screenings by a SHU. This finding indicated that school health services, including vision screenings, have had weaknesses in delivering these services to students for more than three decades. The second major finding related to the role of school support, was that it was not able to detect eye problems earlier. It was found that schools did not cooperate with the school health units, as well as not communicating with the students' parents to provide activities or services that would contribute to detecting eye problems, thus decreasing or preventing these problems among students.

In summary, the findings from this study made several contributions to solving the problem of late detection of visual abnormalities. First, this study suggested increasing the knowledge level of students and parents about eye health services provided by SHUs. Second, the study also suggested that improved school health initiatives would decrease or prevent the prevalence of eye problems.

\section{Implications and Recommendations}

Based on the data analysis and discussion of the present study, there are specific implications and recommendations from the policies related to the role of school health units (SHUs) towards early detecting of correctable visual problems among students. Firstly, to improve the effectiveness and efficiency of current vision screening among students; there is a need for conducting periodic vision screenings by SHUs at least once every semester. In addition, a network of vision care services between schools and SHUs should be established to ensure earlier referral and follow-up procedures. Furthermore, some of the Saudi schools presently have a budget. Thus, they can cooperate with the private sector to conduct vision screenings under the supervision of school health units. Secondly, to decrease the prevalence of ocular disorders among male students in Qassim Province, this study was highly recommended by the importance of a health clinic inside schools. This recommendation can also solve the problem of accessibility and affordability of eye health services. In addition, it can indirectly benefit the health sector by reducing patients overload in hospitals. Although, this recommendation does not coincide the decision of transferring the act of school health units under the supervision of the Ministry of Health $(\mathrm{MOH})$, the study found that health clinics were better to be established in schools rather than transferring the SHUs' activities to the Ministry of Health, because the infrastructure of the $\mathrm{MOH}$ is not ready to receive these units. Accordingly, the Ministry of Education (MOE) should establish health clinics in schools. Thirdly, to increase 
the level of parent knowledge about eye health and help in early detection of eye problems, it is recommended that certain activities should be implemented by schools with the cooperation of SHUs. Such activities include conducting awareness campaigns, and implementing health education sessions in schools. Fourthly, MOE should adopt teaching the curriculum of School Health Education in public schools. Finally, because teachers usually spend most of their school day with students, it is recommended to train them how to detect eye problems. This includes how to conduct vision screening, use screening charts, observe signs or symptoms of visual problems, etc.

\section{Delimitations and Further Research}

Several delimitations narrow the scope of this study. First, the emphasis is on male students. The rules of the Saudi MOE hinder male researchers from conducting studies on female students. Therefore, there was not an opportunity to identify the impact of vision screening of SHUs at early detection among female students. Thereby, the findings of this study may restrict the applicability of the results for female students. Second, the current study is just centre on schools affiliated with two SHUs in Qassim Province to determine the impact of the SHUs' vision screening program and school support in addressing early detection of eye problems among male students. Thus, the findings of this study may not represent the whole SHUs in the province. Finally, as with the female students, there was not an opportunity to identify the impact of the vision screening of the female SHUs at early detection. Therefore, the applicability of the study for female units may be restricted.

The study's findings highlight some important considerations for future research. Firstly, although the findings of this study indicated weakness in the school health units at detecting eye problems earlier, the challenges facing these units should be addressed. In addition, while this study focused on male school health units, there is a need to examine the role of female units. Moreover, in many countries, vision-screening tools have to be developed and enhanced to detect ocular disorders earlier, However, the present study has not examined the validity of vision screening tools. Thus, it was recommended they should be studied. Finally, the current study unveils only the start of vision screening and it is strongly related to early detection. Therefore, this study need to be developed and covered in many of its aspects.

\section{References}

Al-Nasser, A., Sinha, M., \& Ali, M. (1989). Screening of eye health problems amongst school children in Riyadh. Egypt J Comm Med, $5(2), 47-53$.

Al-Rowaily, M. A. (2010). Prevalence of refractive errors among preschool children at King Abdulaziz Medical City, Riyadh, Saudi Arabia. Saudi J Ophthalmol, 24(2), 45-48. doi: 10.1016/j.sjopt.2010.01.001

Al Wadaani, F. A., Amin, T. T., Ali, A., \& Khan, A. R. (2013). Prevalence and pattern of refractive errors among primary school children in Al Hassa, Saudi Arabia. Glob J Health Sci, 5(1), 125-134. doi: 10.5539/gjhs.v5n1p125

Alansari, S. (2001). Entrance to the School Health (second ed.). Riyadh: Khaled Ameen Institution (Arabic refrence).

Aldebasi, Y. H. (2011). Young Public's Awareness to Refractive Error Deficiency. Int J Health Sci (Qassim), 5(1), 9.

Aldebasi, Y. H. (2014). Prevalence of correctable visual impairment in primary school children in Qassim Province, Saudi Arabia. [Research Support, Non-U.S. Gov't]. J Optom, 7(3), 168-176. doi: 10.1016/j.optom.2014.02.001

Aldebasi, Y. H. (2015). Prevalence of amblyopia in primary school children in Qassim province, Kingdom of Saudi Arabia. Middle East Afr J Ophthalmol, 22(1), 86-91. doi: 10.4103/0974-9233.148355

Alotaibi, A. Z. (2006). The state of low vision services in Saudi Arabia. Medical Science and Technology, 47(4), RA215-RA218.

American Academy of Pediatrics. (2001). School health centres and other integrated school health services. Committee on School Health. Pediatrics, 107(1), 198-201.

Arif, M., \& Mehboob, Q. (2014). Visual Screening; Government, Private and Community School going Children in Faisalabad. Professional Med J, 21(6), 112-116.

Baltussen, R., Naus, J., \& Limburg, H. (2009). Cost-effectiveness of screening and correcting refractive errors in school children in Africa, Asia, America and Europe. Health Policy, 89(2), 201-215. doi: 10.1016/j.healthpol.2008.06.003

Bardisi, W. M., \& Bin Sadiq, B. M. (2002). Vision screening of preschool children in Jeddah, Saudi Arabia. Saudi Med J, 23(4), 445-449.

Basch, C. E. (2010). Vision and the achievement gap among urban minority youth. Journal of School Health, 81(10), 599-605.

Biemer, P. P., \& Lyberg, L. E. (2003). Introduction to survey quality (Vol. 335): John Wiley \& Sons.

Desouky, D., E., Murad, N., \& Khan, T. (2014). Refractive error among a sample of Female Primary School Children in Taif City, KSA. International Journal of Public Health and Epidemiology, 3(10), 89-97.

Fotouhi, A., KhabazKhoob, M., Hashemi, H., Yekta, A. A., \& Mohammad, K. (2011). Importance of including refractive error tests in school children's vision screening. Arch Iran Med, 14(4), 250-253. doi: 0011144/aim.005

Fryer, G. E., Igoe, J. B., \& Miyoshi, T. J. (1997). Considering school health program screening services as a cost offset: a comparison of existing reimbursements in one state. J Sch Nurs, 13(2), 18-21.

General Administration of School Health. (2007). Practical Guidebook for a Health Instructor. Riyadh, Saudi Arabia: Ministry of 
Education.

Gupta, A., Lal, R., Mazta, S., \& Sharma, D. (2012). Prevalence of Refractive Errors, Color Vision Defects and Other Ocular Disorders in School-going Children: Primary Screening by School Teachers. Journal of Internation Medical Sciences Academy, 25(4), 223224.

Gursoy, H., Basmak, H., Yaz, Y., \& Colak, E. (2013). Vision screening in children entering school: Eskisehir, Turkey. Ophthalmic Epidemiol, 20(4), 232-238. doi: 10.3109/09286586.2013.808672

Harvey, J., Vaquerano, L., Nolan, L., \& Sonosky, C. (2002). School-based health centres and managed care arrangements: a review of state models and implementation issues: Centre for Health Services Research and Policy, George Washington University.

Hinkley, S., Abata, K., \& Schlotthauer, K. (2014). Michigan Teachers' Opinions on the Efficacy of School Vision Screenings. Optom Vis Perf, 2(4), 160-165.

Jen, W. Y. (2009). Mobile healthcare services in school-based health centre. [Research Support, Non-U.S. Gov't]. Int J Med Inform, 78(6), 425-434. doi: 10.1016/j.jimedinf.2008.11.002

Kemper, A. R., Uren, R. L., \& Clark, S. J. (2006). Barriers to Follow-up Eye Care After Preschool Vision Screening in the Primary Care Setting: Findings From a Pilot Study. Journal of AAPOS: The Official Publication of the American Association for Pediatric Ophthalmology and Strabismus / American Association for Pediatric Ophthalmology and Strabismus, 10(5), 476-478. doi: 10.1016/j.jaapos.2006.07.009

Kimel, L. S. (2006). Lack of follow-up exams after failed school vision screenings: an investigation of contributing factors. J Sch Nurs, 22(3), 156-162

Kisker, E. E., \& Brown, R. S. (1996). Do school-based health centres improve adolescents' access to health care, health status, and risktaking behavior? J Adolesc Health, 18(5), 335-343. doi: 10.1016/1054-139x(95)00236-I

Lear, J. G. (2002). Schools and adolescent health: strengthening services and improving outcomes. J Adolesc Health, 31(6 Suppl), 310320.

Mathers, M., Keyes, M., \& Wright, M. (2010). A review of the evidence on the effectiveness of children's vision screening. Child: care, health and development, 36(6), 756-780. doi: 10.1111/j.1365-2214.2010.01109.x

Murthy, G. (2000). Vision Testing for Refractive Errors in Schools: "Screening" Programmes in Schools. Community Eye Health, 13(33), 3.

Nila Ethel, E., \& Nagalakshmi, P. (2014). Early Intervention Measures for Refractive Error. Indian Journal of Applied Research, 4(8). doi: $10.15373 / 2249555 X$

Powell, C., Wedner, S., \& Richardson, S. (2005). Screening for correctable visual acuity deficits in school-age children and adolescents. Cochrane Database Syst Rev(1), CD005023. doi: 10.1002/14651858.CD005023.pub2

Prasad, K. R. (2005). School health. Indian Journal of Community Medicine, $30(4), 3$.

Qureshi, M. A., Ahmed, M. I., Al-debasi, Y. H., \& Nair, V. (2012). Prevalence of Incidental Amblyopia in Buraidah City. Pakistan Journal of Ophthalmology, 28(3), 141.

Resnikoff, S., Pascolini, D., Mariotti, S. P., \& Pokharel, G. P. (2008). Global magnitude of visual impairment caused by uncorrected refractive errors in 2004. Bulletin of the World Health Organization, 86(1), 63-70.

Rowaily, M. A., \& Alanizi, B. M. (2010). Prevalence of Uncorrected Refractive Errors among Adolescents at King Abdul-Aziz Medical City, Riyadh, Saudi Arabia. Journal of Clinical \& Experimental Ophthalmology, 01(03). doi: 10.4172/2155-9570.1000114

Rushood, A., Azmat, S., Shariq, M., Khamis, A., Lakho, K., Jadoon, M., . . Kamil, E. (2013). Ocular disorders among schoolchildren in Khartoum State, Sudan. Eastern Mediterranean Health Journal, 19(3), 282-288.

Santelli, J., Kouzis, A., \& Newcomer, S. (1996). School-based health centres and adolescent use of primary care and hospital care. J Adolesc Health, 19(4), 267-275. doi: 10.1016/s1054-139x(96)00088-2

Su, Z., Marvin, E. K., Wang, B. Q., van Zyl, T., Elia, M. D., Garza, E. N., . . Forster, S. H. (2013). Identifying barriers to follow-up eye care for children after failed vision screening in a primary care setting. J AAPOS, 17(4), 385-390. doi: 10.1016/j.jaapos.2013.05.008

Taylor, H. R., Pezzullo, M. L., Nesbitt, S. J., \& Keeffe, J. E. (2007). Costs of interventions for visual impairment. Am J Ophthalmol, 143(4), 561-565. doi: 10.1016/j.ajo.2006.10.055

Wedner, S. H., Ross, D. A., Balira, R., Kaji, L., \& Foster, A. (2000). Prevalence of eye diseases in primary school children in a rural area of Tanzania. British journal of ophthalmology, 84(11), 1291-1297.

Wedner. S., \& Dineen. B. (2003). Refractive errors. Tropical Doctor, 33, 9-207.

Woodhouse, J. M., Davies, N., McAvinchey, A., \& Ryan, B. (2014). Ocular and visual status among children in special schools in Wales: the burden of unrecognised visual impairment. [Research Support, Non-U.S. Gov't]. Arch Dis Child, 99(6), 500-504. doi: 10.1136/archdischild-2013-304866 